\title{
Applying ZEKE-spectroscopy to clusters
}

\author{
G.F. Gantefor, D.M. Cox, and A. Kaldor \\ Corporate Research Laboratories, Exxon Research and Engineering Company, Clinton Township, Route 22 East, Annandale, \\ NJ 08801, USA
}

\begin{abstract}
In this paper we describe the application of a special form of electron photodetachment spectroscopy, zero electron kinetic energy (ZEKE) spectroscopy, to the study of cold metal cluster anions. From this study we have obtained vibrational and thus structural information of several neutral and charged clusters, e.g. $\mathrm{Au}_{2}^{-10}, \mathrm{Ag}_{3}^{-10}$, $\mathrm{Sn}_{2}^{-10}$ and $\mathrm{Al}_{3}^{-10}$. In addition, in separate measurements of the relative photodetachment cross section of $\mathrm{Au}_{2}^{-}$, we have discovered sharp resonances which are due to electronic autodetachment from a short lived excited state.
\end{abstract}

PACS: $36.40 .+\mathrm{d} ; 33.60 .-\mathrm{q}$

\section{Introduction}

Photoelectron spectroscopy of negative ions (photodetachment spectroscopy = PDS) is a powerful tool to study the electronic properties of gas phase metal clusters, and to date has served as the primary source of information about the electronic structure of metal cluster anions $[1,2,3]$. However, it is clear that cluster geometry (e.g. vibrational frequencies, potential curves etc.) is an important parameter for which little information is available. Concerning PDS geometric structure must be inferred from a comparison of theoretical electronic structure calculations with experimental results [4].

In order to obtain additional structural information for small clusters we have applied a special form of electron photodetachment spectroscopy, namely zero electron kinetic energy (ZEKE) spectroscopy $[5,6,7]$, to the study of transition metal cluster anions. From such studies we are able to extract information as the vibrational temperature, vibrational frequencies, anharmonicities, Franck-Condon transition probabilities, adiabatic

\footnotetext{
${ }^{1}$ In our apparatus we do not have an electron energy analyser and
} thus are not able to obtain conventional photoelectron spectra. electron affinities etc., and thus structural information. To date we have studied small gold, silver, tin and aluminium cluster anions.

\section{Experimental}

The experimental technique consists of producing a beam of cluster anions, mass separating them and then collecting the electrons detached when a particular size cluster anion is irradiated with a dye laser as described in a recent publication [8]. The main points to note are:

(i) the cluster anion source can produce cold cluster anions $(T \sim 165 \mathrm{~K})$,

(ii) only electrons between 0 and $1.5 \mathrm{meV}$ kinetic energy are detected in the ZEKE mode of operation.

This combination of low temperature anions and high electron energy resolution allows us to obtain electronic and vibrational properties of neutral and charged clusters.

In addition to the ZEKE mode of operation where only the lowest energy electrons are detected, we also can operate in a second mode where nearly all electrons are collected [9]. This mode allows us to measure the relative photodetachment cross section. ${ }^{1}$

In both types of spectroscopy the detaching laser flux has to be kept as low as possible to avoid space charge effects, saturation and power broadening. Typical values are $1-10 \mu \mathrm{J} / \mathrm{cm}^{2}$. By changing the expansion conditions, mainly by altering the delays between pulsed helium nozzle and the vaporization laser, the vibrational temperature can be altered over a limited range from $\sim 165 \mathrm{~K}$ to about room temperature.

\section{Results}

$\mathrm{Au}_{2}^{-}$has been carefully studied in both the ZEKE and the relative cross section modes over the photon energy range $1.85-2.2 \mathrm{eV}$ 
The ZEKE-spectrum of $\mathrm{Au}_{2}^{-}$[8] shows sharp peaks (width $\sim 1.5 \mathrm{meV}$ ) only at photon energies corresponding to the threshold energy of each new vibronic transition. Two vibrational progressions have been identified with a frequency of about $188 \mathrm{~cm}^{-1}$ and $146 \mathrm{~cm}^{-1}$. Changing the expansion conditions alters the relative intensities of some of the transitions allowing hot band transitions from populated ground state vibrational levels of $\mathrm{Au}_{2}^{-}$to be identified.

The dependence of the relative photodetachment cross section on the photon energy [9] is expected to be relatively unstructured shows a rather constant value with steplike increases at wavelengths associated with the opening of new photodetachment channels. In addition we observe several (8) sharp and narrow (width $<1 \mathrm{meV}$ ) peaks resembling a vibrational structure on this relatively smooth cross section. The eight sharp peaks which have been detected occur only in a small energy range $(1.95-2.00 \mathrm{eV})$. By observing the change in the relative intensities of the sharp peaks with changing expansion conditions, we have been able to identify cold bands associated with the ground state of $\mathrm{Au}_{2}^{-}$and a new excited state of $\mathrm{Au}_{2}^{-}$with vibrational frequencies of $149 \mathrm{~cm}^{-1}$ and $128 \mathrm{~cm}^{-1}$, respectively. Thus far, we have found sharp features in the relative photodetachment cross section for $\mathrm{Au}_{2}^{-}$and $\mathrm{Au}_{6}^{-}$.

With ZEKE-spectroscopy we studied $\mathrm{Sn}_{2}^{-}, \mathrm{Cu}_{3}^{-}, \mathrm{Ag}_{3}^{-}$ and $\mathrm{Al}_{3}^{-}$with photon energies close to the electron affinity. As an example, Fig. 1 b shows a ZEKE-spectrum of $\mathrm{Ag}_{3}^{-}$. Only a single narrow symmetric peak centered near $2.452 \mathrm{eV}$ is observed. The FWHM of this peak is less than $10 \mathrm{meV}$ and the onset of the electron signal is about $2.44 \mathrm{eV}$. By adjusting the source parameters to result in less effective cooling, the lineshape becomes slightly asymmetric and the onset shifts towards lower energies. Figure 1a displays a conventional PD-spectrum of $\mathrm{Ag}_{3}^{-}$[2] taken with a fixed frequency laser and an hemispherical analyser. The single feature is asymmetric and much broader than that in Fig. $1 b$.

\section{Discussion}

The vibrational parameters directly obtained from the ZEKE-spectrum of cold $\mathrm{Au}_{2}^{-}$[8] are in good agreement with results of a Franck-Condon fit procedure of a standard PD spectrum of $\mathrm{Au}_{2}^{-}\left(T_{\text {vib }} \sim 350 \mathrm{~K}\right)$ from W.C. Lineberger's group [2]. The ground state frequencies of $\mathrm{Au}_{2}$ and $\mathrm{Au}_{2}^{-}$are $188 \mathrm{~cm}^{-1}$ and $146 \mathrm{~cm}^{-1}$, respectively. Because of the high resolution we were able to resolve the 0-0 transition and determine the adiabatic electron affinity to be $1.940 \mathrm{eV} \pm 0.5 \mathrm{meV}$.

The two different features ( $\mathrm{a}$ and $\mathrm{b}$ above) in the relative photodetachment cross section of $\mathrm{Au}_{2}^{-}$[9] can be understood as the direct process (a) and an interferring resonant process $(b)$ due to an autodetaching state. Changing the vibrational temperature of the anion allows differentiation of hot band transitions and assignment of the lines. The vibrational frequency of this excited negative ion state is $128 \mathrm{~cm}^{-1}$, much lower than the neutral ground state frequency of $188 \mathrm{~cm}^{-1}$. We propose that the

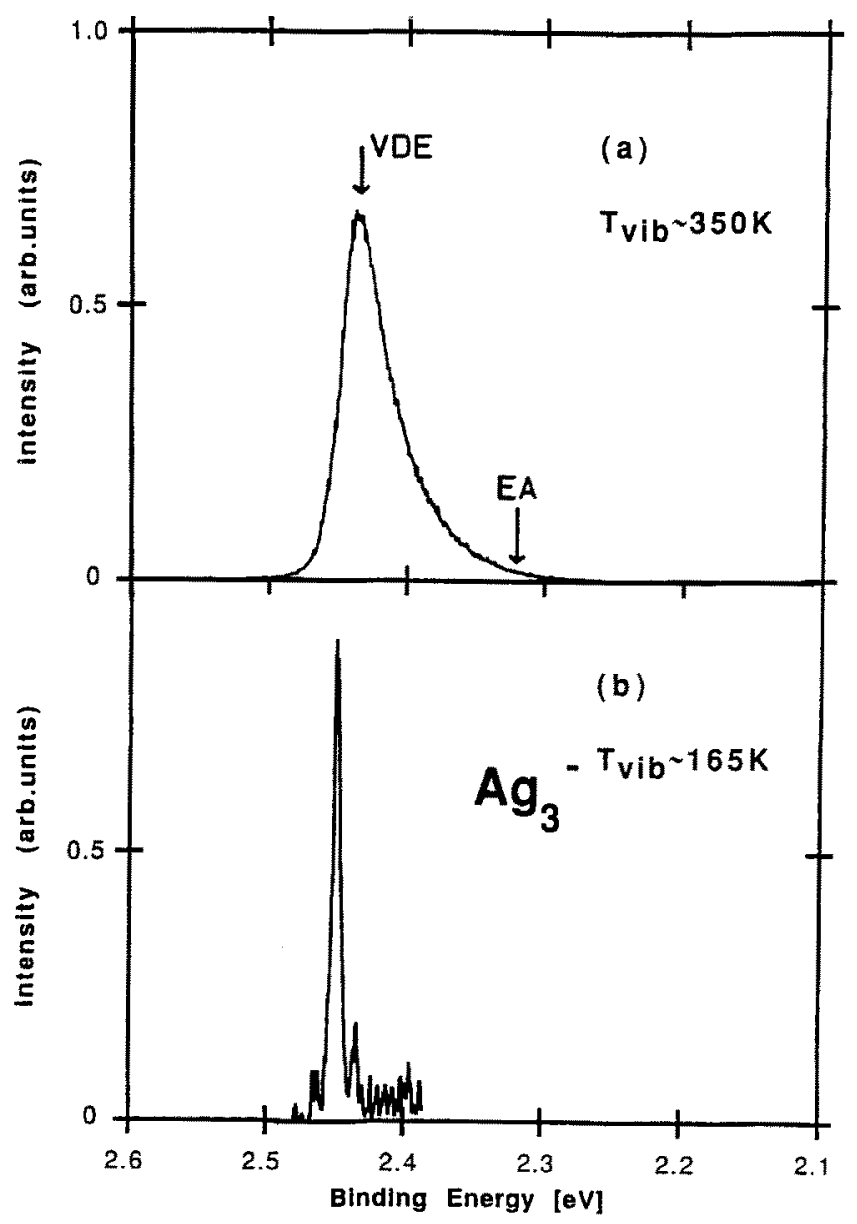

Fig. 1a,b. Comparison of photoelectron spectra of $\mathrm{Ag}_{3}^{-}$taken (a) with a fixed photon energy and a hemispherical analyser to measure the electron kinetic energy $\left(E_{b}=h v-E_{\mathrm{kin}}\right)[2]$ and (b) using ZEKE spectroscopy, i.e. detecting electrons of low kinetic energy $\left(E_{\mathrm{kin}}<1.5 \mathrm{meV}\right)$ only and varying the photon energy $\left(E_{b}=h v\right)$. The vertical detachment energy (VDE) corresponds to the maximum intensity of the feature in a. The electron affinity (EA) is $\sim 100 \mathrm{meV}$ [12] lower than VDE due to the energy difference of the linear and triangular ground state of the neutral (see text). Note the difference in vibrational temperature and positions of the peaks for the two spectra.

resonances are due to electronic autodetachment from an excited state created when a bonding electron is excited into the half-filled non-bonding $\sigma^{*}$ orbital. Detachment occurs when one electron from the $\sigma^{*}$ orbital is ejected while the other relaxes back into the $\sigma$ hole, i.e. an Augerlike process. This is different from the mechanism proposed to explain similar resonances observed recently for $\mathrm{Au}_{6}^{-}[10]$. For $\mathrm{Au}_{6}^{-}$the excited state is proposed to be a weakly bound surface state and as such would have a vibrational frequency similar to the neutral ground state decaying via vibrational autodetachment. We plan to apply ZEKE spectroscopy to $\mathrm{Au}_{6}^{-}$to obtain the $\mathrm{Au}_{6}$ vibrational structure.

A comparison between PDS and ZEKES data as shown in Fig. 1 yields interesting information. Apart from experimental broadening and rotational effects, the main difference in the two types of spectroscopy is the dependency of ZEKES data on the threshold behaviour of the 
photodetachment cross section. If the initial state of the electron possesses some $p$-character the outgoing electron wave can have $s$-character resulting in a step-like onset of photodetachment [11] and then the spectra can be directly compared to PDS-data. This is observed for $\mathrm{Au}_{2}^{-}$in agreement with theoretical data concerning the symmetry of the initial state [8]. In the case of $\mathrm{Ag}_{3}^{-}$the orbital of the outer electron again likely possesses some $p$-character [2]. Thus, the differences of the two spectra shown in Fig. 1a and $b$ must have another explanation.

Theoretical calculations [2] show that the ground state of $\mathrm{Ag}_{3}^{-}$has a linear geometry, while the neutral trimer ground state is triangular. Thus, transitions between the two states are expected to have very low Franck-Condon factors. The lowest linear neutral state is approximately $100 \mathrm{meV}$ above the neutral ground state. This linear state is geometrically similar to the anion ground state resulting in nearly no vibrational excitation due to photodetachment, i.e. a single line. This is strongly supported by our data (Fig. 1b). At higher temperatures transitions into the triangular ground state of the neutral become possible, since bending modes of anion can be populated. In this case we would expect some electron signal towards lower energies with the line broadened asymmetrically towards lower energy, i.e. towards the adiabatic electron affinity (EA). Thus, the differences in the two spectra are caused by temperature effects. The corresponding (known) temperatures of the dimers are included in the figure.

\section{Conclusion}

We have applied zero electron kinetic energy spectroscopy (ZEKES) to study the metal cluster anions $\mathrm{Au}_{2}^{-}$and
$\mathrm{Ag}_{3}^{-}$and have obtained information for the ground electronic states of the neutrais and the anions and the electron affinities. The ability to vary the vibrational temperature of the anions by changing the expansion conditions proved to be invaluable in the assignment of vibrational transitions. Measurements of the relative photodetachment cross section of $\mathrm{Au}_{2}^{-}$results in the discovery of an electronically autodetaching excited state of the anion.

\section{References}

1. Cheshnovsky, O., Taylor, K.J., Conceicao, J., Smalley, R.E.: Phys. Rev. Lett. 64, 1785 (1990)

2. Hoe, J., Ervin, K.M., Lineberger, W.C.: J. Chem. Phys. (1990) (submitted)

3. Gantefoer, G., Gausa, M., Meiwes-Broer, K.H., Lutz, H.O.: Z. Phys. D-Atoms, Molecules and Clusters 12, 405 (1989)

4. Bonacic-Koutecky, V., Fantucci, P., Koutecky, J.: Chem. Phys. Lett. 166, $32(1990)$

5. Mueller-Dethlefs, K., Sander, M., Schlag, E.W.: Z. Naturforsch. 39a, 1089 (1984)

6. Kitsopoulos, T.N, Waller, I.M., Loeser, J.G., Neumark, D.M.: Chem. Phys. Lett. 159, 300 (1989)

7. Harrington, J.E., Weisshaar, J.C.: J. Chem. Phys. 93, 854 (1990)

8. Gantefor, G.F., Cox, D.M., Kaldor, A.: J. Chem. Phys. 93, 8395 (1990)

9. Gantefor, G.F., Cox, D.M., Kaldor, A.: J. Chem. Phys. 94, 854 (1991)

10. Taylor, K.I., Jin, C., Conceicao, J, Lai-Sheng Wang, Cheshnovsky, O., Johnson, B.R., Nordlander, P.J., Smalley, R.E.: J. Chem. Phys. 93, 7515 (1990)

11. Wigner, E.P.: Phys. Rev. 73, 1002 (1948) 